\title{
Clinical applications of resting state functional connectivity
}

\author{
Michael D. Fox ${ }^{*}$ and Michael Greicius ${ }^{2}$ \\ 1 Partners Neurology Residency, Massachusetts General Hospital, Brigham and Women's Hospital, Harvard Medical School, Boston, MA, USA \\ 2 Department of Neurology and Neurological Sciences, Stanford University School of Medicine, Stanford, CA, USA
}

\author{
Edited by: \\ Lucina Q. Uddin, \\ Stanford University, USA \\ Reviewed by: \\ F. Castellanos, \\ Nathan Kline Institute, USA; New York \\ University School of Medicine, USA \\ Yufeng Zang, \\ Beijing Normal University, China \\ *Correspondence: \\ Michael D. Fox, \\ Partners Neurology Residency, \\ Massachusetts General Hospital, \\ Brigham and Women's Hospital, \\ Harvard Medical School, 55 Fruit St., \\ Boston, MA 02114, USA. \\ e-mail: foxmdphd@gmail.com
}

\begin{abstract}
During resting conditions the brain remains functionally and metabolically active. One manifestation of this activity that has become an important research tool is spontaneous fluctuations in the blood oxygen level-dependent (BOLD) signal of functional magnetic resonance imaging (fMRI). The identification of correlation patterns in these spontaneous fluctuations has been termed resting state functional connectivity ( $\mathrm{fCMRI}$ ) and has the potential to greatly increase the translation of $\mathrm{FMRI}$ into clinical care. In this article we review the advantages of the resting state signal for clinical applications including detailed discussion of signal to noise considerations. We include guidelines for performing resting state research on clinical populations, outline the different areas for clinical application, and identify important barriers to be addressed to facilitate the translation of resting state fcMRI into the clinical realm.
\end{abstract}

Keywords: fMRI, fcMRI, neurological disease, psychiatric disease, brain, spontaneous activity, intrinsic activity

\section{INTRODUCTION}

Functional magnetic resonance imaging ( $\mathrm{fMRI}$ ) is a non-invasive technique for examining brain function that utilizes changes in blood oxygen level-dependent (BOLD) signal to identify areas of increased or decreased neuronal activity (Logothetis, 2003; Raichle and Mintun, 2006). This technique has proven extremely valuable in the laboratory environment, allowing researchers to identify brain areas associated with the processing of different stimuli or the performance of various cognitive tasks (Raichle, 2000). Further, fMRI has been used extensively to identify abnormalities in these activation patterns in populations of patients with neurological or psychiatric disease.

Despite its success and popularity as a research tool, fMRI has seen relatively little translation into the clinical realm. In general, the fMRI abnormalities seen in clinical research populations have not translated into the ability to obtain useful diagnostic or prognostic information in individual patients (Matthews et al., 2006). While pre-operative fMRI is being used in individual patients to guide neurosurgical intervention, its use has not yet been shown to improve patient outcomes. Although progress is certainly being made, the clinical utility of fMRI has yet to be firmly established.

A recent advance that offers tremendous promise for improving the clinical applicability of fMRI involves focusing on spontaneous modulations in the BOLD signal that occur during resting conditions (for recent review see Fox and Raichle, 2007). In contrast to the traditional task-based approach, resting state studies observe the brain in the absence of overt task performance or stimulation. In these studies, subjects are generally asked to lie quietly under "resting" conditions such as eyes closed or while fixating on a crosshair. Spontaneous modulations in the BOLD signal in the absence of any explicit input or output are then recorded and analyzed. Although alternative approaches exist (Zhu et al., 2005,
2008; Cao et al., 2006; Fox and Raichle, 2007; Zang et al., 2007; Biswal et al., 2010), analysis of these spontaneous fluctuations usually involves the identification of correlations between remote brain areas, commonly referred to as functional connectivity. The term "functional connectivity" has been used in both resting-state and task-state studies and can refer to correlations across subjects, runs, blocks, trials, or individual BOLD time points, an ambiguity which can become confusing (Friston et al., 1993; Horwitz, 2003; Fox and Raichle, 2007; Rogers et al., 2007). We will therefore use the term resting state functional connectivity MRI (fcMRI) for added specificity, and this will be the focus of the present article. The two most popular techniques for performing resting state fcMRI are seed-based correlations and independent components analysis (ICA). In the seed-based technique signal is extracted from a specific region of interest, and a map is created by computing the correlation between this extracted signal and all other brain voxels (Biswal et al., 1995; Fox and Raichle, 2007). In contrast, ICA considers all voxels at once and uses a mathematical algorithm to separate a dataset into distinct systems or networks that are correlated in their spontaneous fluctuations but also maximally independent, usually in the spatial domain (Kiviniemi et al., 2003; Bartels and Zeki, 2004; Beckmann et al., 2005).

Regardless of the technique, a consistent observation is that regions with similar functional properties, such as the left and right somatomotor cortices, exhibit coherent BOLD fluctuations even in the absence of movement under resting conditions (Biswal et al., 1995; Lowe et al., 1998; Cordes et al., 2000; De Luca et al., 2005; Fox et al., 2006b). Similar results have been found in multiple other networks including visual (Lowe et al., 1998; Cordes et al., 2000), auditory (Cordes et al., 2000), language (Cordes et al., 2000; Hampson et al., 2002), dorsal and ventral attention systems (Fox et al., 2006a), corticothalamic circuits (Zhang et al., 2008), and a frontal opercular network that has been related to stimulus salience 
(Seeley et al., 2007b). One of the most robustly identified and extensively investigated resting state networks involves a set of regions that routinely decrease their activity during attention demanding tasks, often referred to as the default mode network (Raichle et al., 2001; Greicius et al., 2003; Fox et al., 2005; Fransson, 2005) (Figure 1). Interestingly, this network has also been found to be negatively or anti-correlated with regions that tend to increase their activity during attention demanding tasks (Greicius et al., 2003; Fox et al., 2005, 2009; Fransson, 2005; Chang and Glover, 2010). Resting state correlation patterns across various networks have been shown to predict the task-response properties of brain regions (De Luca et al., 2005; Vincent et al., 2006), identify subjects' aptitude for different cognitive tasks (Hampson et al., 2006; Seeley et al., 2007b), and help constrain and refine neuro-anatomical models developed on the basis of task-activation studies (Fox et al., 2006a; Dosenbach et al., 2007).

Given the success of resting state functional connectivity for probing the brain's functional architecture in normal subjects, it is only natural to apply the technique towards understanding brain disease. Two recent reviews detail the large number of studies that have utilized resting state fcMRI to study various neurological and psychiatric conditions (Greicius, 2008; Zhang and Raichle, 2010). Although this list continues to grow on a daily basis, the goal of the present article is not to review the findings from each individual study and the insight each provides towards understanding specific diseases. The field has expanded to the point that resting state reviews focused on each specific disease are rapidly becoming appropriate. Instead, we take a more global perspective on the application of resting state fcMRI in the clinical realm. We detail the theoretical and practical motivations for using resting state fcMRI for clinical applications, describe the different types of clinical applications to

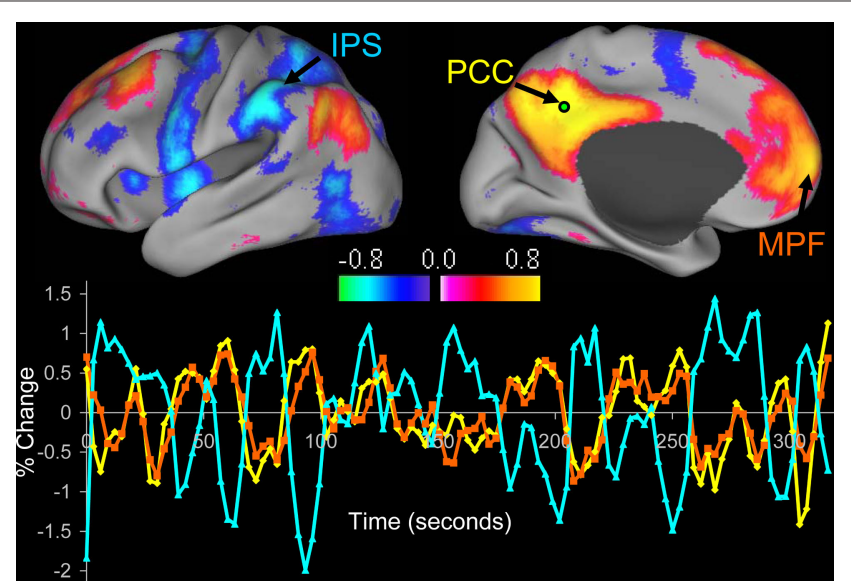

FIGURE 1 | Resting state functional connectivity reveals correlations and anticorrelations with the default mode network. Correlations between a seed region in the posterior cingulate/precuneus (PCC) and all other voxels in the brain for a single subject during resting fixation. Both correlations (positive values) and anticorrelations (negative values) are shown, thresholded at $R=0.3$. The time course for a single run is shown for the seed region (PCC yellow), a region positively correlated with this seed region in the medial prefrontal cortex (MPF, orange), and a region negatively correlated with the seed region in the intraparietal sulcus (IPS, blue). Reproduced with permission from (Fox et al., 2005). which resting state may be applied, provide guidelines for using resting state fcMRI as a clinical tool, and identify barriers to full translation of resting state fcMRI into the clinical realm.

\section{WHY USE RESTING STATE fCMRI FOR CLINICAL APPLICATIONS? \\ CEREBRAL ENERGETICS}

There are several motivations, both theoretical and practical for using resting state fcMRI for clinical applications. The first of these motivations comes from an understanding of brain energy metabolism. The resting human brain represents only $2 \%$ of total body mass but consumes $20 \%$ of the body's energy, most of which is used to support of ongoing neuronal signaling (Ames, 2000; Attwell and Laughlin, 2001; Lennie, 2003; Shulman et al., 2004; Raichle and Mintun, 2006). Task-related increases in neuronal metabolism are usually small $(<5 \%)$ when compared to this large resting energy consumption (Raichle and Mintun, 2006). Differences in these taskrelated changes between normal and pathological populations are smaller still, often less than $1 \%$. When attempting to study disease or diagnose patients based on task-related changes, one is therefore focusing on only a very small fraction of the brain's overall activity. Ongoing spontaneous activity may provide a window onto the neural processing that appears to consume the vast majority of the brain's resources and so may prove a richer source of diseaserelated signal changes.

\section{SIGNAL TO NOISE}

Resting state studies may offer a better signal to noise ratio than conventional task-based approaches. To demonstrate this principle, BOLD modulations recorded from the somatomotor cortex are shown during a simple task in which subjects were asked to press a button with their right hand (Figure 2) (Fox et al., 2006b). In this case, the subject pressed the button only once during the scanning session. Examining the tracing from the left somatomotor cortex alone (Figure 2A), it is impossible to identify when during the session that button press occurred. The signal, or task-related modulation, is very small relative to the tremendous amount of ongoing noise. Even if one focuses only on the time of the button press itself, when task-related BOLD modulation is maximal, the task-related modulation accounts for only $20 \%$ of the total BOLD variance (Fox et al., 2006b, 2007b). This means that during a standard $\mathrm{PMRI}$ task session over $80 \%$ of the BOLD modulation may be discarded as noise. This is why task-related BOLD studies require a large number of trials and extensive averaging to obtain a signal or activation map, and this may be part of the reason why task-based fMRI has found only limited application in the clinical realm.

A critical observation that forms the basis of resting state fcMRI was the finding that much of this "noise" that is so problematic for task-based studies is actually ongoing spontaneous fluctuations that are correlated within distinct cortical networks. This becomes apparent in our button press example when one adds the tracing from the right somatomotor cortex, which is only minimally involved in the right-handed task, to the tracing already shown for the left somatomotor cortex (Figure 2B). Much of the "noise" in the left somatomotor cortex is also present on the right. It is important to note that this shared variance is specific 
to the somatomotor system and can be directly tied to variability in motor function (Fox et al., 2006b, 2007b). Even if one focuses only on the button press epoch, spontaneous ongoing activity can account for around 60\% of the BOLD "noise" (Fox et al., 2006b, 2007b). In fact, one can subtract the ongoing spontaneous activity from the left somatomotor cortex and the single button press response becomes evident (Figure 2C).

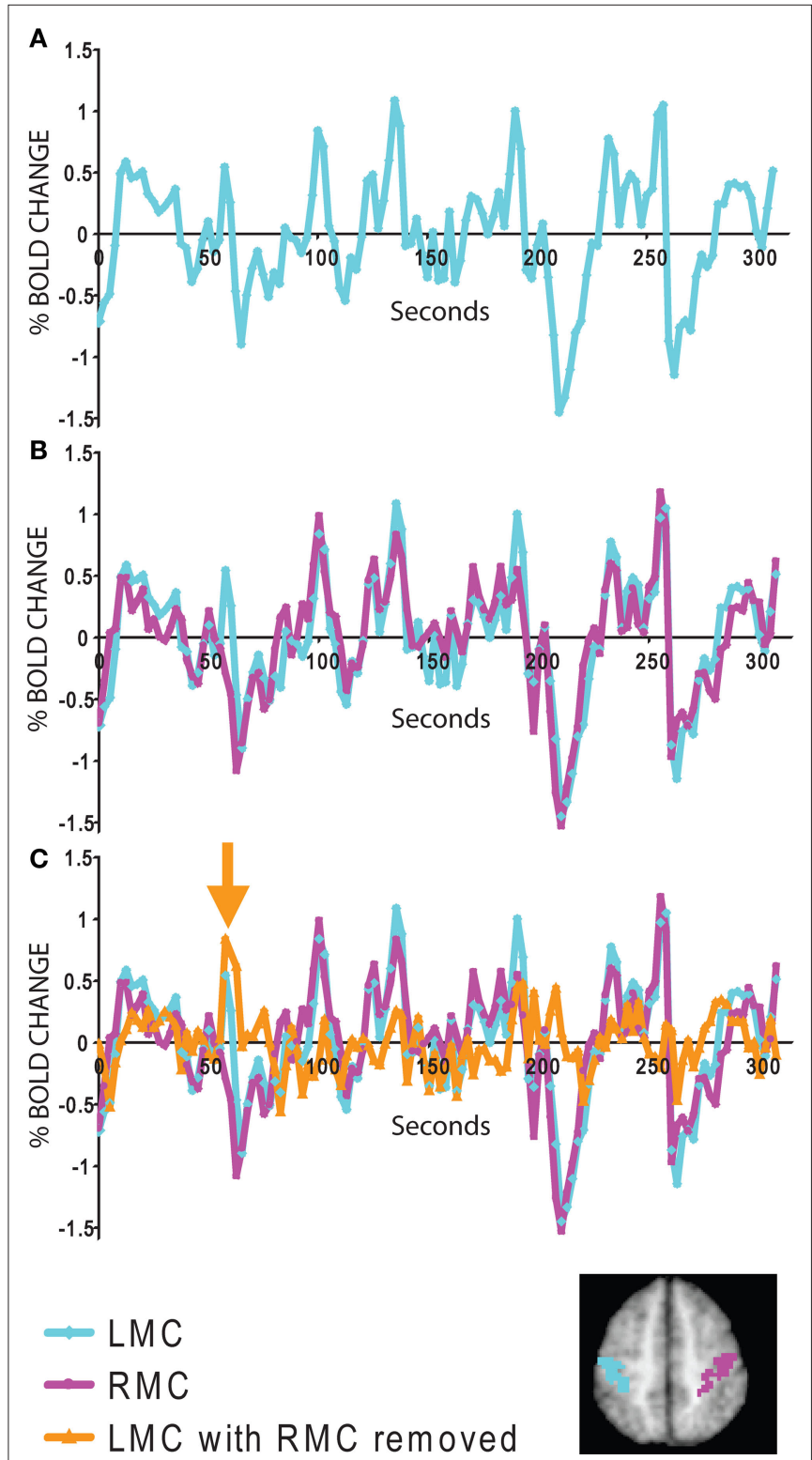

FIGURE 2 | Signal to noise features of spontaneous and task evoked activity. (A) fMRI time course from the left somatomotor cortex (LMC) during a single run when the subject pressed the button once with his right hand. Due to poor signal to noise, it is impossible to identify the task-related activity. (B) Comparison of the LMC with the right somatomotor cortex (RMC) shows that much of the noise is ongoing spontaneous activity correlated within the somatomotor system. (C) After subtracting the RMC from the LMC, the task-related modulation from the individual button press is evident (orange arrow). The LMC and RMC regions of interest are displayed for convenience on the inset map. Data taken from (Fox et al., 2006b).
Task-activation studies have a poor signal to noise ratio because the signal (task-related modulation) is often small relative to the sea of ongoing noise (including spontaneous activity). In contrast, resting state fcMRI focuses on this ongoing spontaneous activity and uses it as the signal rather than discarding it as noise. Systemspecific correlation values can be as high as $0.7-0.9$ (accounting for $50-80 \%$ of the variance) (Fox et al., 2006b, 2007b) (see Figure 1). Compared to the $20 \%$ signal to noise ratio seen in task-based activation studies, resting state fcMRI studies may enjoy approximately three times the signal to noise ratio. Although additional signal to noise considerations exist (see final section), a 3 to 1 improvement in signal to noise has obvious advantages when attempting to identify imaging abnormalities in individual patients.

\section{MULTI-PURPOSE DATA SETS}

In addition to the above signal to noise considerations, resting state fcMRI data sets can be used to study multiple cortical systems. This is in contrast to task-activation analyses which require dedicated data acquisitions for each function one is attempting to localize. For example, if one wants to identify both motor and language systems for pre-operative mapping one would need to perform one acquisition of a motor task and another acquisition of a language task. In fcMRI the same data can be used to examine both systems, effectively doubling the amount of data (or alternatively reducing the acquisition time by half).

\section{EXPANDED PATIENT POPULATIONS}

One of the most frequently cited motivations for using resting state fcMRI in clinical studies is that it allows for a broader sampling of patient populations. Due to cognitive dysfunction or physical impairment many patients are simply not capable of performing tasks accurately in the fMRI scanner. When studying disease, this often means that we are sampling the least impaired subjects in a patient group as opposed to the most impaired subjects likely to show the largest signal abnormalities. In addition to limiting our sensitivity for detecting disease related changes, this introduces the problem of whether observed abnormalities can be generalized to the average (and often sicker) disease population. Resting state fcMRI requires no task and places only minimal demands on the patient. Further, spontaneous activity continues when subjects are asleep (Fukunaga et al., 2006; Horovitz et al., 2006) and sedated (Kiviniemi et al., 2003; Peltier et al., 2005; Vincent et al., 2007; Greicius et al., 2008b) opening up the possibility of obtaining resting state activity in any patient population. Of note, it remains unclear if individual differences observed during the awake state persist during sleep or sedation and is an important area for future research.

\section{CIRCUMVENTING TASK-RELATED CONFOUNDS}

One important advantage of resting state fcMRI is that it may circumvent confounds that can complicate interpretation of taskbased studies. For example, working memory tasks have been used extensively to study patients with schizophrenia. However, a difference in activation between patients and control subjects observed during the task could represent differences in task performance, effort, task strategy, or an underlying disease-specific brain abnormality. A second example involves longitudinal studies 
which utilize repeated task-based scanning sessions to examine drug effects or disease progression. These repeated task sessions can be confounded by practice effects or adaptation to the task. By eliminating the task, resting state fcMRI can circumvent some of these interpretative ambiguities and may allow for identification of more fundamental abnormalities underlying disease.

\section{TYPES OF CLINICAL APPLICATIONS IDENTIFYING GROUP DIFFERENCES IN BRAIN DISEASE}

Although there are several ways in which resting state fcMRI may be applied to clinical populations, by far the largest application has been comparing resting state correlation patterns between groups of normal subjects and those with neurological or psychiatric disease (for recent reviews see Fox and Raichle, 2007; Greicius, 2008; Zhang and Raichle, 2010). The goal is that through identification of group differences one may begin to better understand the functional abnormalities underlying different disease states leading ultimately to a reliable resting state fcMRI marker that can be interpreted at the single subject level. This knowledge could in turn lead to identification of new treatments or drug targets. Disturbances in the correlation structure of spontaneous activity have now been reported for a significant number of disease states (see Table 1).

The goal of the current review is not to detail the individual findings of over 60 publications across 20 disease states. As mentioned in the introduction, we are rapidly approaching the point where reviews of resting state abnormalities for each particular disease state are becoming appropriate. However, tabulating the studies in this manner does lead to a few important observations. First, resting state fcMRI studies have been published on almost all major neurological and psychiatric diseases as well as a number of related conditions. While replication plays a role, the novelty of simply comparing correlation patterns between two groups is subsiding, and the route is paved for more advanced analyses (see next section). Second, the consistency of resting state abnormalities various greatly by disease state, from excellent consistency across Alzheimer's, MCI, and PIB-positive patients to inconsistent and occasionally opposing findings in schizophrenia. There may be several reasons for this heterogeneity, and some mechanism to reconcile disparate findings is needed. Third, a seemingly disproportionate number of studies seem to focus on the default mode network as opposed to other resting state networks. While this may be appropriate in diseases like Alzheimer's with known or theoretical pathology in these regions, some of this focus may stem from a misconception that the default mode network is somehow special in showing large-amplitude coherent BOLD fluctuations at rest (for additional discussion see Fox and Raichle, 2007; Zhang and Raichle, 2010).

\section{OBTAINING DIAGNOSTIC AND PROGNOSTIC INFORMATION}

Given the substantial group comparison literature now available (Table 1), the route is paved for more advanced analyses of resting state abnormalities. One important advance is to relate the resting state differences seen between two groups to a relevant clinical variable. For example, pathological disturbances in intrinsic activity have been correlated with the severity of disease in depression (Greicius et al., 2007), schizophrenia (Bluhm et al., 2007), and neglect (He et al., 2007). Relating resting state abnormalities to a relevant clinical variable speaks directly to the potential clinical relevance of a given finding and greatly increases confidence that the reported resting state abnormality will be reproducible.

Another important advance towards identifying prognostic or diagnostic markers on individual patients is to calculate the ability of observed resting state abnormalities to segregate healthy from disease states. Not surprisingly, the vast majority of this work has focused on the disease state with the most reproducible resting state abnormalities, Alzheimers, (Li et al., 2002; Greicius et al., 2004; Wang et al., 2006a; Supekar et al., 2008) (Figure 3) and the potentially associated condition of PIB positivity (Hedden et al., 2009; Sheline et al., 2010). By looking at different resting state fcMRI measures and setting a threshold, one can calculate the sensitivity and specificity of that marker for segregating healthy and disease states (Figure 3A). In Alzheimers, sensitivity ranges from $72-85 \%$ and specificity from 77-80\% (Li et al., 2002; Greicius et al., 2004; Wang et al., 2006a; Supekar et al., 2008). Instead of picking just one threshold, receiver operating characteristic (ROC) curves can show the sensitivity and specificity at several different thresholds and have been usefully applied in Alzheimers (Li et al., 2002; Supekar et al., 2008) (Figures 3B,C). Although not yet applied to Alzheimers, techniques such as machine vector learning and advanced pattern recognition may further improve the utility of resting state fcMRI abnormalities as brain disease biomarkers (Haynes and Rees, 2006; Norman et al., 2006). Thus far, these segregation studies have been retrospective and the criteria for identifying a disease has been optimized for a specific data set. Future work applying these criteria prospectively towards new datasets will serve as an important test of their potential clinical relevance as a diagnostic or prognostic marker.

\section{LONGITUDINAL STUDIES AND TREATMENT EFFECTS}

One area for which resting state fcMRI is extremely well suited is longitudinal studies and monitoring treatment effects. For example, much may be learned by following disease progression in neuro-degenerative disorders such as Alzheimer's or amyotrophic lateral sclerosis (ALS). Similarly, one can examine the effect of clinical intervention by studying subjects before and after treatment. Normalization of resting state brain abnormalities with drug therapy may prove to be a useful surrogate outcome in clinical trials or help pharmaceutical companies decide which drugs to bring to large-scale clinical trials in the first place. Along these lines, resting state fcMRI abnormalities in depression have been shown to dissipate with drug treatment (Anand et al., 2005b), and improvement in regional correlations has been shown to match functional recovery in spatial neglect following stroke (He et al., 2007).

\section{CLUSTERING IN HETEROGENEOUS DISEASE STATES}

To date segregation has focused largely on differentiating healthy from disease states. However one important role for resting state connectivity analyses may be segregating patients within a disease category. For example, schizophrenia is widely regarded as a very heterogeneous disorder, and this heterogeneity can greatly hinder the sensitivity of clinical trials. One could imagine placing the resting state patterns of hundreds of patients with schizophrenia into an algorithm that would cluster the patients into groups 
Table 1 | Group differences in resting state fcMRI patterns observed in various brain diseases or conditions.

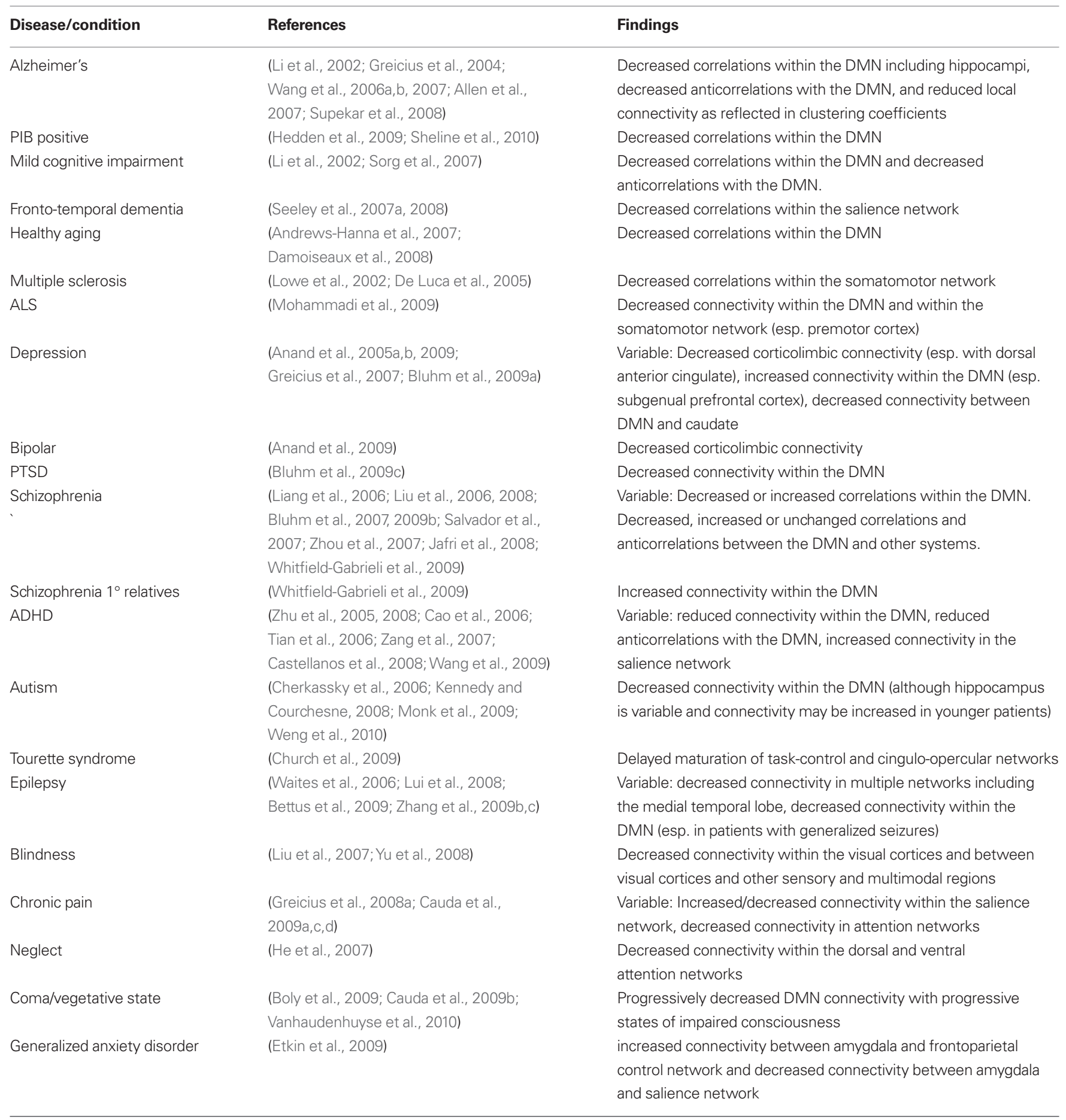

$D M N=$ default mode network including regions in the posterior cingulate/precuneus, lateral parietal cortex, medial temporal lobes, and medial prefrontal cortex (see Figure 1). Salience network: includes regions in the dorsal anterior cingulate and bilateral fronto/insular cortices; dACC = dorsal anterior cingulated cortex; $P I B=$ Pittsburg compound B, a marker of amyloid plaque accumulation in the brain. PTSD = post-traumatic stress disorder; ALS = amyotrophic lateral sclerosis; $A D H D$ = attention deficit hyperactivity disorder. Note: some references (Greicius et al., 2004; He et al., 2007) reflect "near-rest" conditions in which task-related variance has been minimized and other references (Zhu et al., 2005, 2008; Cao et al., 2006; Zang et al., 2007) reflect local changes in spontaneous BOLD fluctuations as opposed to correlations in these fluctuations between separate regions.

with similar resting state abnormalities. Similarly, retrospective analysis of drug effects could identify subgroups that benefited from a particular therapy.

\section{PRE-OPERATIVE MAPPING AND TARGETING INTERVENTION}

The area in which traditional task-based fMRI has shown the greatest promise for clinical translation is in pre-operative functional 


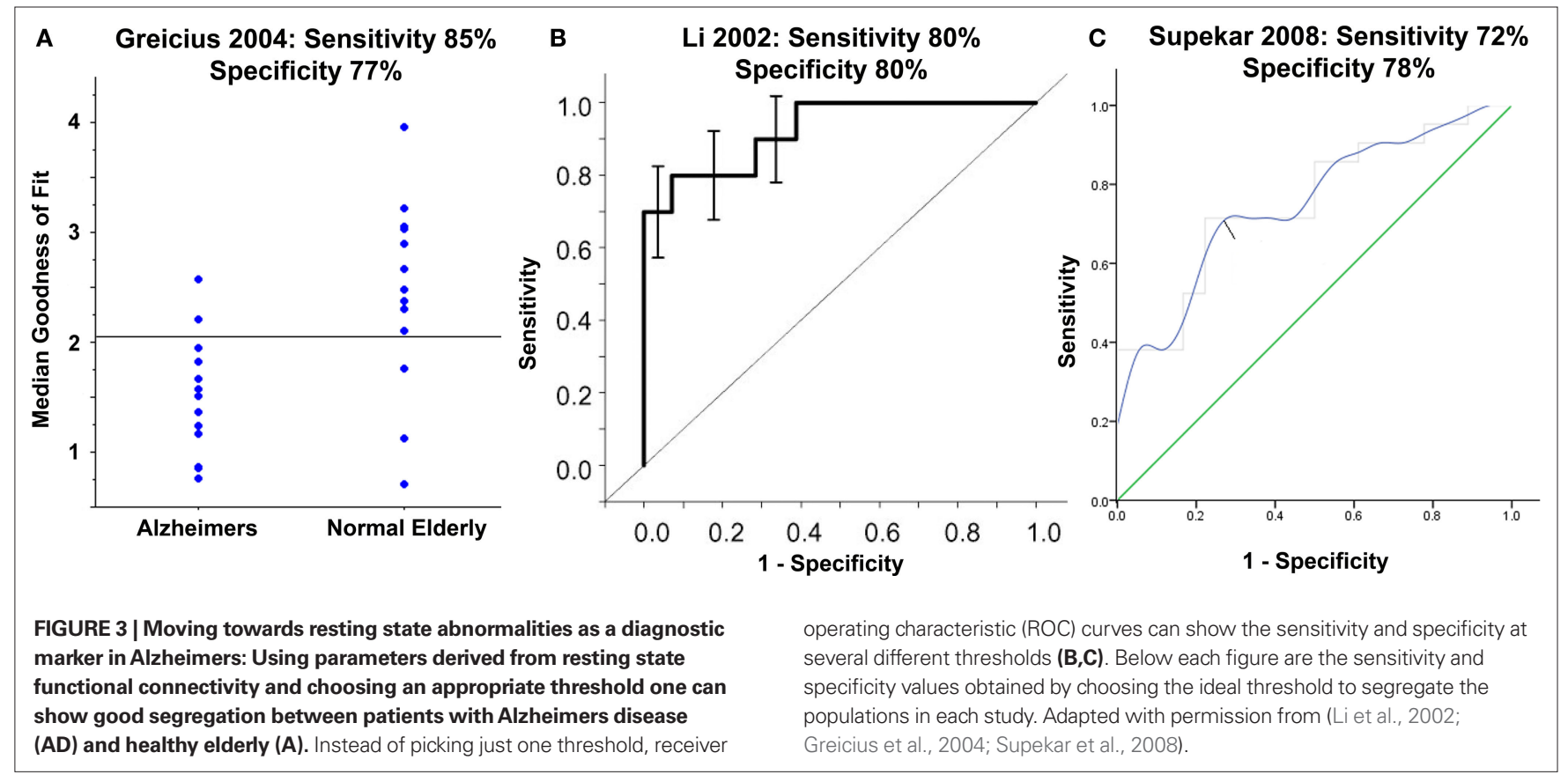

brain mapping to help guide neurosurgical planning (Haberg et al., 2004; Vlieger et al., 2004; Matthews et al., 2006). This is used most often to identify brain areas used in movement and language so that these areas can be avoided during surgical resection, but it has also been combined with EEG to identify foci of epileptic activity (Lemieux, 2004). fMRI defined brain regions correlate with intraoperative electrophysiology (Vlieger et al., 2004), Wada testing (Binder et al., 1996; Adcock et al., 2003), loss-of-function postoperatively (Haberg et al., 2004), and are frequently mentioned in neurosurgery notes (Haberg et al., 2004). However, patients frequently lack the ability to perform tasks well (Pujol et al., 1998) and patient movement during tasks can be a significant problem (Lee et al., 1999).

As mentioned earlier, the advantages of resting state fMRI may circumvent many of the current limitations hindering task-based pre-operative mapping. Indeed several articles have recently been published showing strong proof of concept for resting state fcMRI as a pre-operative mapping tool in patients with neurosurgical conditions (Kokkonen et al., 2009; Liu et al., 2009; Shimony et al., 2009; Zhang et al., 2009a). These articles have shown good correlation between resting state fcMRI results, task-based mapping, and intraoperative cortical stimulation in these patients (Figure 4).

Just as resting state fcMRI may guide surgeons in their operative approach, it may also be used to guide several other clinical interventions where localization of a functional region is critical. Examples include placement of EEG recording grids, deep brain stimulators, and transcranial magnetic stimulation (TMS).

\section{BARRIERS TO CLINICAL APPLICABILITY/FUTURE WORK GUIDELINES FOR STUDYING CLINICAL POPULATIONS WITH fcMRI}

Despite the promise of resting state fcMRI for improving the translation of functional imaging into the clinical realm, several challenges remain. One of the largest barriers is inconsistent
Table 2 | Guidelines for studies of clinical populations with resting state fcMRI.

(1) A priori hypotheses regarding a region or network with abnormal fcMRI and clear criteria for selecting this region or network

(2) A priori hypothesis and demonstration of a region or network with normal fcMRI to serve as a control

(3) Correlation with clinical variables whenever possible

(4) Stringent correction for multiple comparisons

(5) An analysis of movement in patients and control subjects

(6) An analysis of the differential impact of pre-processing in patients and control subjects

(7) A discussion of how current findings relate to prior fcMRI findings

results across studies. When studies are relatively consistent, as in Alzheimers, it is easy to build on these results and move towards using resting state fcMRI for diagnostic and prognostic purposes. However, when studies are inconsistent as in schizophrenia, one is left wondering which result, if any, is most likely to be reproducible and therefore clinically relevant. Different study designs, processing techniques, analysis approaches, and regions or systems of interest make comparing studies very difficult.

One of the first steps towards improving translation is to begin to improve our ability to replicate and compare results from different resting state studies. While individual labs will always differ in their analytical approach (and this is a good thing) there are certain standards or guidelines that may help improve reproducibility and strengthen the conclusions that can be made (Table 2). Some of these guidelines may appear generic and obvious, however resting state fcMRI presents a unique set of challenges in study design and analysis that may benefit from explicit delineation. 


\section{^ \\ Structural MRI}

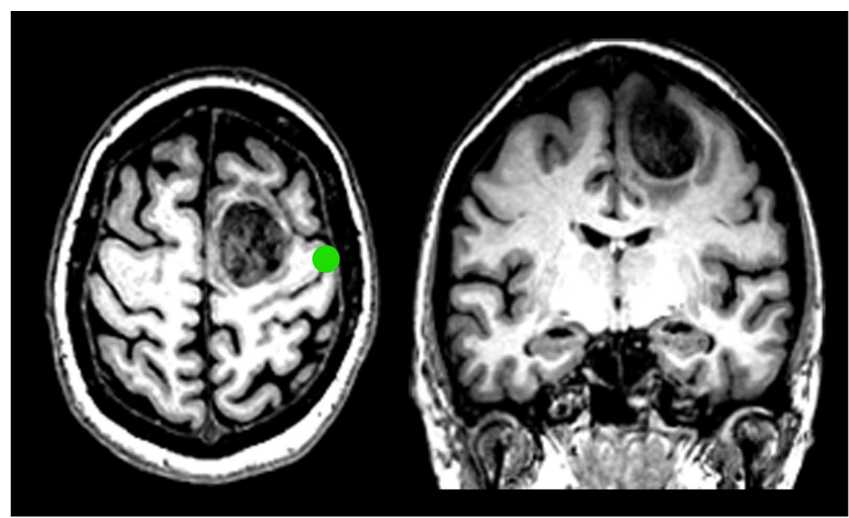

$z=64 \quad y=-12$

B

\section{Task \\ Evoked}

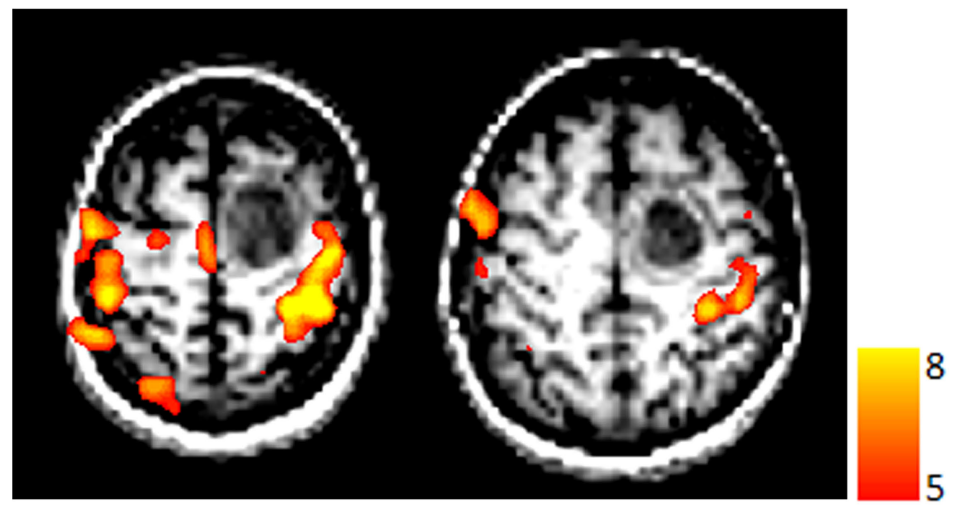

$$
z=66 \quad z=54
$$

C

\section{Resting State}

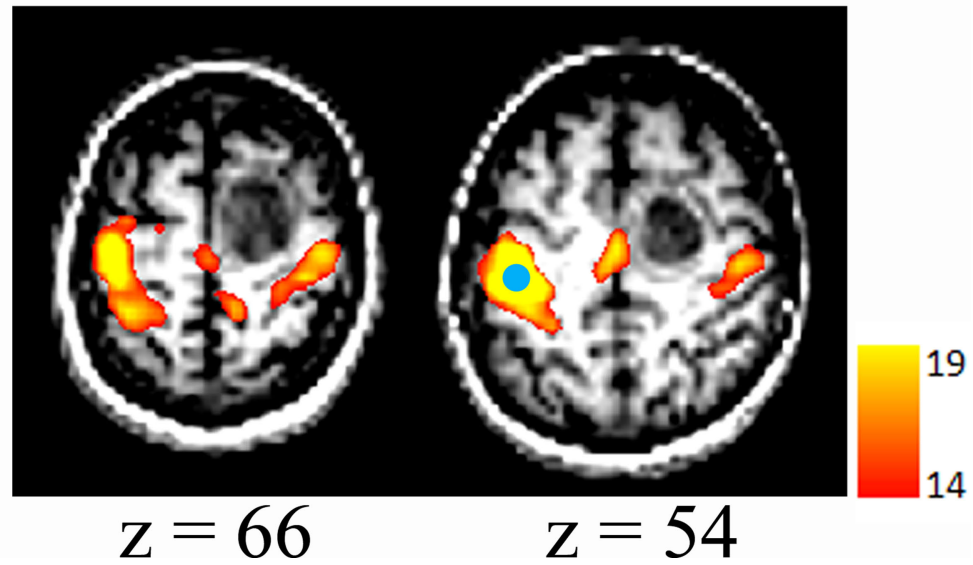

FIGURE 4 | Resting state fcMRI in pre-operative brain mapping:

(A) Structural MRI scan showing a mass in the right frontal cortex. Green circle represents the location of ipsilateral hand response to intra-operative cortical stimulation. (B) Task-related mapping showing activity within the sensorimotor network but also small responses in parietal cortex that are seemingly

(1) The first guideline concerns a priori identification of either a region (seed-based analysis) or network (ICA) that one expects may be abnormal. This hypothesis can be based on prior imaging data (either task-based or resting state), pathology, or simply the clinical features of the disease combined unrelated to motor function or sensation. (C) Resting-state correlation mapping showing that the sensorimotor network is largely unaffected by the tumor anterior to the central sulcus. Seed region is shown (blue circle). All images are displayed left-on-left. Adapted with permission from (Zhang et al., 2009a).

with theory suggesting localization of the relevant impaired functions. If the a priori motivation for the study is clearly presented in the introduction, then even a well-powered, negative finding can represent an advance. Analyses of a large number of seed regions or components can be an effective 
means of generating hypotheses, but such exploratory work must be followed by targeted analyses that are powered to disconfirm spurious findings. Similar to choosing which region or network one is interested in, one must also clearly identify a priori how that network will be identified. If one is using a seed region, the coordinates for that seed region should be justified, for example as a focus of activation from a previously published study. Similarly, if one is studying a network in the form of an ICA component, one needs to specify an objective approach for identifying that system such as spatial correlation to an a priori template (Greicius et al., 2004, 2007), however see also (Zuo et al., 2009) for possible limitations of this approach.

(2) Perhaps as important as the first guideline, the second guideline involves a priori identification of regions or networks that one expects NOT to vary between the disease and healthy state. A good choice for many diseases may be primary sensory systems such as visual, somatomotor, or auditory. Of course, it is theoretically possible that a disease state exists which impacts every brain system and region such that a normal control is not possible. However in these cases alternative control strategies should be pursued to show that the findings are not artifactual.

(3) As mentioned in an earlier section, any study which can show a relationship between identified resting state fcMRI abnormalities and clinical variables such as disease severity increases the confidence that a finding will be clinically relevant and reproducible.

(4) The fourth guideline concerns correction for multiple comparisons. This becomes especially pertinent if one is looking for differences across a large number of seed regions or components or if one is attempting to correlate resting state abnormalities with several different clinical variables. The probability of finding a significant relationship increases as the number of variables one is trying to relate increases. Several methods to correct for these multiple comparisons exist, the simplest and most stringent being Bonferroni correction (Abdi, 2007). Clearly there are cases where one doesn't know a priori which clinical variable or component may be of interest, and an effect that does not pass Bonferroni does not mean the effect is not interesting, it simply means that the relationship would benefit from repeat and targeted testing.

(5) The fifth guideline concerns movement correction and comes from the recognition that patient populations are often going to be less cooperative lying in the scanner than control populations, especially when they are required to do nothing but stare at a fixation cross for $10 \mathrm{~min}$. While task-based studies can partially compensate for movement by averaging across a large number of trials, the nature of the signal used in resting state makes it particularly susceptible to movement confounds. Movement parameters are often used as co-regressors in resting state fMRI to try to minimize artificial correlations, however if large group differences in movement are present this remains a confounding variable. In such instances, one could look to see if movement correlated on a subject to subject basis with the finding of interest. If the patients that moved the most also showed the largest difference in resting state correlation values then there should be an elevated index of suspicion. Note that identification of control networks that are not different between the two groups will also help in this regard (see point \#2).

(6) Similar to the above movement analysis, one should examine the impact of pre-processing on the two groups of subjects to insure that they do not differ. For example, much has been written on the pronounced effect of global signal regression on resting state correlations and anticorrelations (Chang and Glover, 2009, 2010; Fox et al., 2009; Murphy et al., 2009; Weissenbacher et al., 2009). Although there is benefit to this pre-processing maneuver including improved correspondence with anatomical connectivity (Fox et al., 2009), one must ensure that the effect of the pre-processing was not different in the two groups. In this example, one could examine the variance removed by global regression and show that it is not significantly different between patients and controls. If there is a group difference then one may want to repeat the analysis without removing the global signal and determine if the effect of interest remains. Similarly in ICA, there is a large impact on results based on the number of components one chooses. Due to a difference in variance in a patient population from movement or any other confounding factor, a certain component could be split at a different point in patients and controls. Repeating a finding with a slightly higher or lower number of components (such as plus and minus $25 \%$ of the initial number of components) could increase confidence in the result.

(7) The final guideline concerns reconciling findings with previously published work. Although this point may seem obvious and is certainly not specific to resting state fcMRI, its importance makes it worth mentioning. If the current resting state fcMRI findings conflict with prior fcMRI work, it is crucial to explore possible etiologies of the conflict. It should not be sufficient to simply mention that other work has been done with differing conclusions. Resolving the discrepancy may involve additional analyses to directly explore differences in processing methodology, but such analyses are critical for accelerating consensus in the field and clinical applicability.

\section{THE CASE FOR COLLABORATION}

Despite the increasing number of papers being published on a daily basis by individual labs, clinical applicability of fcMRI is not likely to move forward without enhanced collaboration and data sharing between labs. Different processing techniques for analyzing resting state data make comparison across studies difficult. The majority of resting state articles focus on a few seed regions or a single network, leaving unexplored the vast majority of the brain's functional architecture. Finally, almost all studies focus on normal subjects or a single disease population making it difficult to assess reproducibility or determine the sensitivity or specificity of an identified abnormality for a specific disease.

In this review we explored several factors that make resting state fcMRI well-suited for translation into the clinical realm. However there are also several features that make it well-suited for databasing, data sharing, and collaboration. Due to the nature of spontaneous BOLD data, a single dataset can be used for multiple analyses and can address a wide variety of neuroscience questions. Furthermore, the paradigms used to study spontaneous BOLD 
activity are relatively simple compared to task-based imaging studies with multiple stimuli presented at varying intervals. These factors make spontaneous BOLD data ideally suited for reanalysis and inclusion in a database.

The above factors have motivated the creation of two online databases focused on resting state fcMRI data. The first is both an analysis package and database termed BrainSCAPE (Spontaneous Correlation Analysis Processing Engine) ${ }^{1}$ (Fox et al., 2007a). This tool allows users to upload, analyze, and share their spontaneous BOLD data as well as analyze freely shared data from other labs. More recently a second database has been launched termed the NITRIC 1000 connectome project $^{2}$ and includes a large number of functional connectivity datasets freely available for download (Biswal et al., 2010). By providing access to multiple datasets, effects in one study can easily be confirmed and compared with results from multiple other datasets. We anticipate that collaborative projects such as these will accelerate advances in the field and may prove valuable in assessing the sensitivity and specificity of intrinsic abnormalities underlying human disease.

\section{TECHNIQUE DEVELOPMENT}

Finally, an improvement in clinical utility is likely to come from further technique development. One area that is likely to be essential as we move from studies of groups of patients to obtaining prognostic and diagnostic information on a single patient is improving signal to noise. As mentioned at the beginning of this article, studies of resting state fluctuations do enjoy a potential signal to noise advantage over task-based studies. However, in task-based studies one can improve the signal to noise by simply increasing the number of trials and the amount of averaging. The technique for improving signal to noise in resting state studies is less straight forward. It is important to recognize that not all spontaneous BOLD fluctuations are due to underlying neuronal fluctuations in distinct cortical systems but may also come from non-neuronal sources. Although the quantitative impact of these noise sources is likely small relative to neuronal fluctuations, spontaneous BOLD modulation can be measured in a water phantom (Zarahn et al., 1997), and physiological fluctuations such as cardiac or respiratory activity can account for a significant fraction of spontaneous BOLD variance in human data (Glover et al., 2000; Wise et al., 2004; Birn et al., 2006; Lund et al., 2006; Chang and Glover, 2009). Improvements in signal to noise could therefore come from reducing the contribution of these non-neuronal fluctuations.

One strategy to account for non-neuronal noise is to employ a high sampling rate which prevents aliasing of higher frequency cardiac or respiratory activity (Biswal et al., 1995; Lowe et al., 1998; Cordes et al., 2001; De Luca et al., 2006); however this comes

${ }^{1}$ www.brainscape.org

${ }^{2}$ www.nitrc.org/projects/fcon_1000

\section{REFERENCES}

Abdi, H. (2007). "Bonferroni and Šidák corrections for multiple comparisons," in Encyclopedia of Measurement and Statistics, ed. N. J. Salkind (Thousand Oaks, CA: Sage).

Adcock, J. E., Wise, R. G., Oxbury, J. M., Oxbury, S. M., and Matthews, P. M. (2003). Quantitative fMRI assessment

with the limitation of reduced spatial coverage. Alternatively, physiological parameters can be measured during BOLD acquisition and removed from the data through linear regression (Glover et al., 2000; Rombouts et al., 2003; Birn et al., 2006; Deshpande et al., 2006; Lund et al., 2006; Chang et al., 2009; Chang and Glover, 2009). Finally, noise sources can be isolated from the BOLD data itself through techniques such as ICA (Kiviniemi et al., 2003; Bartels and Zeki, 2004; Beckmann et al., 2005), regressing out signals common to all voxels (the global signal) (Zarahn et al., 1997; Macey et al., 2004; Fox et al., 2005, 2009), or regressing out signals from regions likely to have a relatively high degree of physiological artifact relative to the amount of neuronal activity such as the ventricles or white matter (Rombouts et al., 2003; Fox et al., 2005). By improving signal to noise, one can begin to reduce scan time and improve clinical applicability.

Other technique advances that may be helpful are increasing the fMRI data that can be used for resting state analyses. For example, research may be expanded by using resting epochs from block design task data (Fair et al., 2007) or removing task-related variance and performing fcMRI analyses on the residual (Arfanakis et al., 2000; Fair et al., 2007; He et al., 2007).

Finally, improved clinical applicability will likely come from moving beyond the fMRI scanner to multimodal investigations of spontaneous activity. Spontaneous fluctuations in the BOLD signal have been shown to correlate with EEG (Laufs et al., 2003), local field potentials (Shmuel and Leopold, 2008), and slow cortical potentials recorded with subdural electrode grids (He et al., 2008). Also resting state functional connectivity analyses are now being done with spontaneous fluctuations observed with near infrared spectroscopy (White et al., 2009). Such techniques raise the potential for studying continuous resting state correlations in situations where an MRI scanner is not practical such as real-time monitoring in intensive care units or operating rooms.

\section{CONCLUSIONS}

Resting state fluctuations in the BOLD signal of fMRI provide good signal to noise, require minimal patient compliance, can be obtained under anesthesia, and are well suited for translation into the clinical realm. Clinical applications include research studies focused on group differences, biomarkers for obtaining diagnostic and prognostic information in a single subject, and guidance of invasive and non-invasive treatments. Several guidelines for resting state studies of brain disease have been proposed here and may improve the reproducibility of findings and facilitate clinical translation. Finally, improvement in processing techniques of the fMRI signal as well moving beyond the fMRI signal to other modalities that can also assess low-frequency fluctuations are likely to be important as we begin to realize the potential of resting state fluctuations in the clinical realm.

hippocampal functional connectivity in Alzheimer disease. Arch. Neurol. 64, 1482-1487.

Ames, A. I. (2000). CNS energy metabolism as related to function. Brain Res. Rev. 34, 42-68.

Anand, A., Li, Y., Wang, Y., Lowe, M. J., and Dzemidzic, M. (2009). Resting state corticolimbic connectivity abnormalities in unmedicated bipolar disorder and unipolar depression. Psychiatry Res. 171, 189-198.

Anand, A., Li, Y., Wang, Y., Wu, J., Gao, S., Bukhari, L., Mathews, V. P., Kalnin, A., and Lowe, M. J. (2005a). Activity and connectivity of brain mood regulating circuit in depression: a functional magnetic 
resonance study. Biol. Psychiatry 57, 1079-1088.

Anand, A., Li, Y., Wang, Y., Wu, J., Gao, S., Bukhari, L., Mathews, V. P., Kalnin, A., and Lowe, M. J. (2005b). Antidepressant effect on connectivity of the mood-regulating circuit: an fMRI study. Neuropsychopharmacology 30, 1334-1344.

Andrews-Hanna, J. R., Snyder, A. Z., Vincent, J. L., Lustig, C., Head, D., Raichle, M. E., and Buckner, R. L. (2007). Disruption of large-scale brain systems in advanced aging. Neuron 56 , 924-935.

Arfanakis, K., Cordes, D., Haughton, V. M., Moritz, C. H., Quigley, M. A., and Meyerand, M. E. (2000). Combining independent component analysis and correlation analysis to probe interregional connectivity in fMRI task activation datasets. Magn. Reson. Imaging 18, 921-930.

Attwell, D., and Laughlin, S. B. (2001). An energy budget for signaling in the grey matter of the brain. J. Cereb. Blood Flow Metab. 21, 1133-1145.

Bartels, A., and Zeki, S. (2004). The chronoarchitecture of the human brain - natural viewing conditions reveal a time-based anatomy of the brain. Neuroimage 22, 419-433.

Beckmann, C. F., DeLuca, M., Devlin, J. T., and Smith, S.M. (2005). Investigations into resting-state connectivity using independent component analysis. Philos. Trans. R. Soc. Lond., B, Biol. Sci. 360, 1001-1013.

Bettus, G., Guedj, E., Joyeux, F., ConfortGouny, S., Soulier, E., Laguitton, V., Cozzone, P. J., Chauvel, P., Ranjeva, J. P., Bartolomei, F., and Guye, M. (2009). Decreased basal fMRI functional connectivity in epileptogenic networks and contralateral compensatory mechanisms. Hum. Brain Mapp. 30, 1580-1591.

Binder, J. R., Swanson, S. J., Hammeke, T. A., Morris, G. L., Mueller, W. M., Fischer, M., Benbadis, S., Frost, J. A., Rao, S. M., and Haughton, V. M. (1996). Determination of language dominance using functional MRI: a comparison with the Wada test. Neurology 46, 978-984.

Birn, R. M., Diamond, J. B., Smith, M. A., and Bandettini, P.A. (2006). Separating respiratory-variation-related fluctuations from neuronal-activity-related fluctuations in fMRI. Neuroimage 31, 1536-1548.

Biswal, B., Yetkin, F., Haughton, V., and Hyde, J. (1995). Functional connectivity in the motor cortex of resting human brain using echo-planar MRI. Magn. Reson. Med. 34, 537-541.

Biswal,B.B., Mennes, M.,Zuo,X.N., Gohel, S., Kelly, C., Smith, S. M., Beckmann, C. F., Adelstein, J. S., Buckner, R. L.,
Colcombe, S., Dogonowski, A. M., Ernst, M., Fair, D., Hampson, M., Hoptman, M. J., Hyde, J. S., Kiviniemi, V. J., Kotter, R., Li, S. J., Lin, C. P., Lowe, M. J., Mackay, C., Madden, D. J., Madsen, K. H., Margulies, D. S., Mayberg, H. S., McMahon, K., Monk, C. S., Mostofsky, S. H., Nagel, B. J., Pekar, J. J., Peltier, S. J., Petersen, S. E., Riedl, V., Rombouts, S. A., Rypma, B., Schlaggar, B. L., Schmidt, S., Seidler, R. D., Siegle, G. J., Sorg, C., Teng, G. J., Veijola, J., Villringer, A., Walter, M., Wang, L., Weng, X. C., Whitfield-Gabrieli, S., Williamson, P., Windischberger, C., Zang, Y.F., Zhang, H. Y., Castellanos, F. X., and Milham, M.P. (2010). Toward discovery science of human brain function. Proc. Natl. Acad. Sci. U.S.A. 107, 4734-4739.

Bluhm, R., Williamson, P., Lanius, R., Theberge, J., Densmore, M., Bartha, R., Neufeld, R., and Osuch, E. (2009a). Resting state default-mode network connectivity in early depression using a seed region-of-interest analysis: decreased connectivity with caudate nucleus. Psychiatry Clin. Neurosci. 63, 754-761.

Bluhm, R. L., Miller, J., Lanius, R. A., Osuch, E. A., Boksman, K., Neufeld, R. W., Theberge, J., Schaefer, B., and Williamson, P. C. (2009b). Retrosplenial cortex connectivity in schizophrenia. Psychiatry Res. 174, 17-23.

Bluhm, R. L., Williamson, P. C., Osuch, E. A., Frewen, P. A., Stevens, T. K., Boksman, K., Neufeld, R. W., Theberge, J., and Lanius, R.A. (2009c). Alterations in default network connectivity in posttraumatic stress disorder related to early-life trauma. J. Psychiatry Neurosci. 34, 187-194.

Bluhm, R. L., Miller, J., Lanius, R. A., Osuch, E. A., Boksman, K., Neufeld, R., Theberge, J., Schaefer, B., and Williamson, P. (2007). Spontaneous low-frequency fluctuations in the BOLD signal in schizophrenic patients: anomalies in the default network. Schizophr. Bull. 33, 1004-1012.

Boly, M., Tshibanda, L., Vanhaudenhuyse, A., Noirhomme, Q., Schnakers, C., Ledoux, D., Boveroux, P., Garweg, C., Lambermont, B., Phillips, C., Luxen, A., Moonen, G., Bassetti, C., Maquet, P., and Laureys, S. (2009). Functional connectivity in the default network during resting state is preserved in a vegetative but not in a brain dead patient. Hum. Brain Mapp. 30, 2393-2400.

Cao, Q., Zang, Y., Sun, L., Sui, M., Long, X., Zou, Q., and Wang, Y. (2006). Abnormal neural activity in children with attention deficit hyperactivity disorder: a resting-state functional magnetic resonance imaging study. Neuroreport 17, 1033-1036.
Castellanos, F. X., Margulies, D. S., Kelly, C., Uddin, L. Q., Ghaffari, M., Kirsch, A., Shaw, D., Shehzad, Z., Di Martino, A., Biswal, B., Sonuga-Barke, E. J., Rotrosen, J., Adler, L. A., and Milham, M. P. (2008). Cingulate-precuneus interactions: a new locus of dysfunction in adult attention-deficit/hyperactivity disorder. Biol. Psychiatry 63 332-337.

Cauda, F., D’Agata, F., Sacco, K., Duca, S. Cocito, D., Paolasso, I., Isoardo, G., and Geminiani, G. (2009a). Altered resting state attentional networks in diabetic neuropathic pain. J. Neurol. Neurosurg. Psychiatry. [Epub ahead of print].

Cauda, F., Micon, B. M., Sacco, K., Duca, S., D’Agata, F., Geminiani, G., and Canavero, S. (2009b). Disrupted intrinsic functional connectivity in the vegetative state. J. Neurol. Neurosurg. Psychiatr. 80, 429-431.

Cauda, F., Sacco, K., D’Agata, F., Duca, S. Cocito, D., Geminiani, G., Migliorati, F., and Isoardo, G. (2009c). Lowfrequency BOLD fluctuations demonstrate altered thalamocortical connectivity in diabetic neuropathic pain. BMC Neurosci. 10, 138.

Cauda, F., Sacco, K., Duca, S., Cocito, D., D’Agata, F., Geminiani, G. C., and Canavero, S. (2009d). Altered resting state in diabetic neuropathic pain. PLoS ONE 4, e4542. doi: 10.1371/ journal.pone.0004542.

Chang, C., Cunningham, J. P., and Glover, G.H. (2009). Influence of heart rate on the BOLD signal: the cardiac response function. Neuroimage 44, 857-869.

Chang, C., and Glover, G. H. (2009). Effects of model-based physiological noise correction on default mode network anti-correlations and correlations. Neuroimage 47, 1448-1459.

Chang, C., and Glover, G. H. (2010). Time-frequency dynamics of restingstate brain connectivity measured with fMRI. Neuroimage 50, 81-98.

Cherkassky, V. L., Kana, R. K., Keller, T. A., and Just, M. A. (2006). Functional connectivity in a baseline resting-state network in autism. Neuroreport 17 , 1687-1690.

Church, J. A., Fair, D. A., Dosenbach, N. U., Cohen, A. L., Miezin, F. M. Petersen, S. E., and Schlaggar, B. L. (2009). Control networks in paediatric Tourette syndrome show immature and anomalous patterns of functional connectivity. Brain 132, 225-238.

Cordes, D., Haughton, V. M., Arfanakis K., Wendt, G. J., Turski, P. A., Moritz, C. H., Quigley, M. A., and Meyerand, M. E. (2000). Mapping functionally related regions of brain with functional connectivity MR imaging. Am. J. Neuroradiol. 21, 1636-1644.

Cordes, D., Haughton, V. M., Arfanakis, K., Wendt, G. J., Turski, P. A., Moritz,
C. H., Quigley, M. A., and Meyerand, M.E. (2001). Frequencies contributing to functional connectivity in the cerebral cortex in 'resting-state' data. Am. J. Neuroradiol. 22, 1326-1333.

Damoiseaux, J.S., Beckmann, C.F., Arigita, E. J., Barkhof, F., Scheltens, P., Stam, C. J., Smith, S. M., and Rombouts, S. A. (2008). Reduced resting-state brain activity in the "default network" in normal aging. Cereb. Cortex. 18, 1856-1864.

De Luca, M., Beckmann, C. F., De Stefano, N., Matthews, P. M., and Smith, S. M. (2006). fMRI resting state networks define distinct modes of long-distance interactions in the human brain. Neuroimage 29, 1359-1367.

De Luca, M., Smith, S. M., De Stefano, N., Federico, A., and Matthews, P. M. (2005). Blood oxygenation level dependent contrast resting state networks are relevant to functional activity in the neocortical sensorimotor system. Exp. Brain Res. 167, 587-594.

Deshpande, G., LaConte, S., Peltier, S., and $\mathrm{Hu}, \mathrm{X}$. (2006). Tissue specificity of nonlinear dynamics in baseline fMRI. Magn. Reson. Med. 55, 626-632.

Dosenbach, N. U., Fair, D. A., Miezin, F. M., Cohen, A. L., Wenger, K. K., Dosenbach, R. A. T., Fox, M. D., Snyder, A. Z., Vincent, J. L., Raichle, M. E., Schlagger, B. L., and Petersen, S. E. (2007). Distinct brain networks for adaptive and stable task control in humans. Proc. Natl. Acad. Sci. U.S.A. 104, 11073-11078.

Etkin, A., Prater, K. E., Schatzberg, A. F., Menon, V., and Greicius, M. D. (2009). Disrupted amygdalar subregion functional connectivity and evidence of a compensatory network in generalized anxiety disorder. Arch. Gen. Psychiatry 66, 1361-1372.

Fair, D. A., Schlaggar, B. L., Cohen, A. L., Miezin, F. M., Dosenbach, N. U., Wenger, K. K., Fox, M. D., Snyder, A. Z., Raichle, M. E., and Petersen, S. E. (2007). A method for using blocked and event-related fMRI data to study "resting state" functional connectivity. Neuroimage 35, 396-405.

Fox, M. D., Corbetta, M., Snyder, A. Z., Vincent, J. L., and Raichle, M. E. (2006a). Spontaneous neuronal activity distinguishes human dorsal and ventral attention systems. Proc. Natl. Acad. Sci. U.S.A. 103, 10046-10051.

Fox, M. D., Snyder, A. Z., Zacks, J. M., and Raichle, M.E.(2006b). Coherent spontaneous activity accounts for trial-totrial variability in human evoked brain responses. Nat. Neurosci. 9, 23-25.

Fox,M.D., Marcus, D.M.,Snyder,A.Z., and Raichle, M. E. (2007a). "BrainSCAPE: an online spontnaeous correlation analysis processing environment for 
fMRI BOLD data," in Organization for Human Brain Mapping Annual Meeting, Chicago, IL.

Fox, M. D., Snyder, A. Z., Vincent, J. L., and Raichle, M. E. (2007b). Intrinsic fluctuations within cortical systems account for intertrial variability in human behavior. Neuron 56, 171-184.

Fox, M. D., and Raichle, M. E. (2007). Spontaneous fluctuations in brain activity observed with functional magnetic resonance imaging. Nat. Rev. Neurosci. 8, 700-711.

Fox, M. D., Snyder, A. Z., Vincent, J. L., Corbetta, M., Van Essen, D. C., and Raichle, M. E. (2005). The human brain is intrinsically organized into dynamic, anticorrelated functional networks. Proc. Natl. Acad. Sci. U.S.A. 102, 9673-9678.

Fox, M. D., Zhang, D., Snyder, A. Z., and Raichle, M.E. (2009). The global signal and observed anticorrelated resting state brain networks. J. Neurophysiol. 101, 3270-3283.

Fransson, P. (2005). Spontaneous lowfrequency BOLD signal fluctuations: an fMRI investigation of the restingstate default mode of brain function hypothesis. Hum. Brain Mapp. 26, 15-29.

Friston, K. J., Frith, C. D., Liddle, P. F., and Frackowiak, R.S. J. (1993). Functional connectivity: the principal component analysis of large (PET) data sets. J. Cereb. Blood Flow Metab. 13, 5-14.

Fukunaga, M., Horovitz, S. G., Van Gelderen, P., de Zwart, J. A., Jansma, J. M., Ikonomidou, V. N., Chu, R., Deckers, R. H. R., Leopold, D. A., and Duyn, J. H. (2006). Large-amplitude, spatially correlated fluctuations in BOLD fMRI signals during extended rest and light sleep. Magn. Reson. Imaging 24, 979-992.

Glover, G. H., Li, T.Q., and Ress, D. (2000). Image-based method for retrospective correction of physiological motion artifacts in fMRI: RETROICOR. Magn. Reson. Med. 44, 162-167.

Greicius, M. (2008). Resting-state functional connectivity in neuropsychiatric disorders. Curr. Opin. Neurol. 21, 424-430.

Greicius, M. D., Barad, M., Ueno, T., and Mackey, S. C. (2008a). "Chronic pain remodels the brain's salience network: a resting-state fMRI study," in 14th International Meeting of the Organization for Human Brain Mapping Melbourne, Australia.

Greicius, M. D., Kiviniemi, V., Tervonen, O., Vainionpaa, V., Alahuhta, S., Reiss, A. L., and Menon, V. (2008b). Persistent default-mode network connectivity during light sedation. Hum. Brain Mapp. 29, 839-847.
Greicius, M. D., Flores, B. H., Menon, V., Glover, G. H., Solvason, H. B., Kenna, H., Reiss, A. L., and Schatzberg, A. F. (2007). Resting-state functional connectivity in major depression: abnormally increased contributions from subgenual cingulate cortex and thalamus. Biol. Psychiatry 62, 429-437.

Greicius, M. D., Krasnow, B., Reiss, A. L., and Menon, V. (2003). Functional connectivity in the resting brain: a network analysis of the default mode hypothesis. Proc. Natl. Acad. Sci. U.S.A. 100, 253-258.

Greicius, M. D., Srivastava, G., Reiss, A. L., and Menon, V. (2004). Defaultmode network activity distinguishes Alzheimer's disease from healthy aging: evidence from functional MRI. Proc. Natl. Acad. Sci. U.S.A. 101, 4637-4642.

Haberg, A., Kvistad, K.A., Unsgard, G., and Haraldseth, O. (2004). Preoperative blood oxygen level-dependent functional magnetic resonance imaging in patients with primary brain tumors: clinical application and outcome. Neurosurgery 54, 902-914; discussion 914-905.

Hampson, M., Driesen, N. R., Skudlarski, P., Gore, J. C., and Constable, R. T. (2006). Brain connectivity related to working memory performance. $J$. Neurosci. 26, 13338-13343.

Hampson, M., Peterson, B. S., Skudlarski, P., Gatenby, J.C., and Gore, J.C. (2002). Detection of functional connectivity using temporal correlations in MR images. Hum. Brain Mapp. 15, 247-262.

Haynes, J. D., and Rees, G. (2006). Decoding mental states from brain activity in humans. Nat. Rev. Neurosci. 7, 523-534.

He, B. J., Snyder, A. Z., Vincent, J. L., Epstein, A., Shulman, G. L., and Corbetta, M. (2007). Breakdown of functional connectivity in frontoparietal networks underlies behavioral deficits in spatial neglect. Neuron 53, 905-918.

He, B. J., Snyder, A. Z., Zempel, J. M., Smyth, M. D., and Raichle, M. E. (2008). Electrophysiological correlates of the brain's intrinsic large-scale functional architecture. Proc. Natl. Acad. Sci. U.S.A. 105, 16039-16044.

Hedden, T., Van Dijk, K. R., Becker, J. A., Mehta, A., Sperling, R. A., Johnson, K. A., and Buckner, R. L. (2009). Disruption of functional connectivity in clinically normal older adults harboring amyloid burden. J. Neurosci. 29, 12686-12694.

Horovitz, S. G., Fukunaga, M., de Zwart, J. A., Van Gelderen, P., Fulton, S. C., Balkin, T. J., and Duyn, J. H. (2006). "The default-mode network connectivity during awake and early sleep: a simultaneous EEG-BOLD-fMRI study," in Organization for Human Brain Mapping Annual Meeting, Florence, Italy $686 \mathrm{M}-\mathrm{PM}$.

Horwitz, B. (2003). The elusive concept of brain connectivity. Neuroimage 19 , 466-470.

Jafri, M. J., Pearlson, G. D., Stevens, M. and Calhoun, V. D. (2008). A method for functional network connectivity among spatially independent restingstate components in schizophrenia. Neuroimage 39, 1666-1681.

Kennedy, D. P., and Courchesne, E. (2008). The intrinsic functional organization of the brain is altered in autism. Neuroimage 39, 1877-1885.

Kiviniemi, V., Kantola, J. H., Jauhiainen, J., Hyvarinen, A., and Tervonen, $\mathrm{O}$ (2003). Independent component analysis of nondeterministic fMRI signal sources. Neuroimage 19, 253-260.

Kokkonen, S. M., Nikkinen, J., Remes, J., Kantola, J., Starck, T., Haapea, M., Tuominen, J., Tervonen, O., and Kiviniemi, V. (2009). Preoperative localization of the sensorimotor area using independent component analysis of resting-state fMRI. Magn. Reson. Imaging 27, 733-740.

Laufs, H., Krakow, K., Sterzer, P., Eger, E., Beyerle, A., Salek-Haddadi, A., and Kleinschmidt, A. (2003) Electroencephalographic signatures of attentional and cognitive default modes in spontaneous brain activity fluctuations at rest. Proc. Natl. Acad. Sci. U.S.A. 100, 11053-11058.

Lee, C. C., Ward, H. A., Sharbrough, F. W., Meyer, F. B., Marsh, W. R., Raffel, C., So, E. L., Cascino, G. D., Shin, C., $\mathrm{Xu}, \mathrm{Y}$., Riederer, S. J., and Jack, C. R., Jr. (1999). Assessment of functional MR imaging in neurosurgical planning. AJNR Am. J. Neuroradiol. 20, 1511-1519.

Lemieux,L. (2004).Electroencephalographycorrelated functional MR imaging studies of epileptic activity. Neuroimaging Clin. N. Am. 14, 487-506.

Lennie, P. (2003). The cost of cortical computation. Curr. Biol. 13, 493-497.

Li, S. J., Li, Z., Wu, G., Zhang, M. J., Franczak, M., and Antuono, P. G. (2002). Alzheimer disease: evaluation of a functional MR imaging index as a marker. Radiology 225, 253-259.

Liang, M., Zhou, Y., Jiang, T., Liu, Z., Tian, L., Liu, H., and Hao, Y. (2006). Widespread functional disconnectivity in schizophrenia with resting-state functional magnetic resonance imaging. Neuroreport 17, 209-213.

Liu, H., Buckner, R. L., Talukdar, T., Tanaka, N., Madsen, J. R., and Stufflebeam, S. M. (2009). Task-free presurgical mapping using functional magnetic resonance imaging intrinsic activity. $J$. Neurosurg. 111, 746-754.
Liu, H., Liu, Z., Liang, M., Hao, Y., Tan, L., Kuang, F., Yanhong, Y., Xu, L., and Jiang, T. (2006). Decreased regional homogeneity in schizophrenia: a resting state functional magnetic resonance imaging study. Neuroreport $17,19-22$.

Liu, Y., Liang, M., Zhou, Y., He, Y., Hao, Y., Song, M., Yu, C., Liu, H., Liu, Z. and Jiang, T. (2008). Disrupted smallworld networks in schizophrenia. Brain. 131(Pt 4), 945-961.

Liu, Y., Yu, C., Liang, M., Li, J., Tian, L., Zhou, Y., Qin, W., Li, K., and Jiang, T. (2007). Whole brain functional connectivity in the early blind. Brain. 130(Pt 8), 2085-2096.

Logothetis, N. K. (2003). The underpinnings of the BOLD functional magnetic resonance imaging signal. $J$. Neurosci. 23, 3963-3971.

Lowe, M. J., Mock, B. J., and Sorenson, J. A. (1998). Functional connectivity in single and multislice echoplanar imaging using resting-state fluctuations. Neuroimage 7, 119-132.

Lowe, M. J., Phillips, M. D., Lurito, J. T., Mattson, D. L., Dzemidzic, M., and Mathews, V. P. (2002). Multiple sclerosis: low-frequency temporal blood oxygen level-dependent fluctuations indicate reduced functional connectivity - initial results. Radiology 224, 184-192.

Lui, S., Ouyang, L., Chen, Q., Huang, X., Tang, H., Chen, H., Zhou, D., Kemp G. J., and Gong, Q. (2008). Differential interictal activity of the precuneus/ posterior cingulate cortex revealed by resting state functional MRI at $3 \mathrm{~T}$ in generalized vs. partial seizure. J. Magn . Reson. Imaging 27, 1214-1220.

Lund, T. E., Madsen, K. H., Sidaros, K., Luo, W., and Nichols, T. E. (2006). Non-white noise in fMRI: does modeling have an impact? Neuroimage 29, 54-66.

Macey, P. M., Macey, K. E., Kumar, R., and Harper, R. M. (2004). A method for the removal of global effects from fMRI time series. Neuroimage 22, 360-366.

Matthews, P. M., Honey, G. D., and Bullmore, E. T. (2006). Applications of fMRI in translational medicine and clinical practice. Nat. Rev. Neurosci. 7 , 732-744.

Mohammadi, B., Kollewe, K., Samii, A., Krampfl, K., Dengler, R., and Munte, T. F. (2009). Changes of resting state brain networks in amyotrophic lateral sclerosis. Exp. Neurol. 217, 147-153.

Monk, C. S., Peltier, S. J., Wiggins, J. L., Weng, S. J., Carrasco, M., Risi, S., and Lord, C. (2009). Abnormalities of intrinsic functional connectivity in autism spectrum disorders. Neuroimage 47, 764-772.

Murphy, K., Birn, R. M., Handwerker, D. A., Jones, T. B., and Bandettini, P. A. 
(2009). The impact of global signal regression on resting state correlations: are anti-correlated networks introduced? Neuroimage 44, 893-905.

Norman, K. A., Polyn, S. M., Detre, G. J., and Haxby, J.V. (2006). Beyond mindreading: multi-voxel pattern analysis of fMRI data. Trends Cogn. Sci. (Regul. Ed.) $10,424-430$.

Peltier, S. J., Kerssens, C., Hamann, S. B., Sebel, P. S., Byas-Smith, M., and $\mathrm{Hu}, \mathrm{X}$. (2005). Functional connectivity changes with concentration of sevoflurane anaesthesia. Neuroreport $16,285-288$

Pujol, J., Conesa, G., Deus, J., LopezObarrio, L., Isamat, F., and Capdevila, A. (1998). Clinical application of functional magnetic resonance imaging in presurgical identification of the central sulcus. J. Neurosurg. 88, 863-869.

Raichle, M. E. (2000). "A brief history of human functional brain mapping," in Brain Mapping The Systems, eds A. W. Toga and J. C. Mazziotta (San Diego: Academic Press), 33-75.

Raichle, M.E., MacLeod, A. M., Snyder,A. Z., Powers, W. J., Gusnard, D. A., and Shulman, G.L. (2001). A default mode of brain function. Proc. Natl. Acad. Sci. U.S.A. 98, 676-682.

Raichle, M. E., and Mintun, M. A. (2006). Brain work and brain imaging. Annu. Rev. Neurosci. 29, 449-476.

Rogers, B. P., Morgan, V. L., Newton, A. T., and Gore, J. C. (2007). Assessing functional connectivity in the human brain by fMRI. Magn. Reson. Imaging $25,1347-1357$.

Rombouts, S. A. R. B., Stam, C. J., Kuijer, J. P. A., Scheltens, P., and Barkhof, F. (2003). Identifying confounds to increase specificity during a "no task condition". Evidence for hippocampal connectivity using fMRI. Neuroimage 20, 1236-1245.

Salvador, R., Martinez, A., PomarolClotet, E., Sarro, S., Suckling, J., and Bullmore, E. (2007). Frequency based mutual information measures between clusters of brain regions in functional magnetic resonance imaging. Neuroimage $35,83-88$.

Seeley, W. W., Allman, J. M., Carlin, D. A., Crawford, R. K., Macedo, M. N., Greicius, M. D., Dearmond, S. J., and Miller, B. L. (2007a). Divergent social functioning in behavioral variant frontotemporal dementia and Alzheimer disease: reciprocal networks and neuronal evolution. Alzheimer Dis. Assoc. Disord. 21, S50-57.

Seeley, W. W., Menon, V., Schatzberg, A. F., Keller, J., Glover, G. H., Kenna, H., Reiss, A. L., and Greicius, M. D. (2007b). Dissociable intrinsic connectivity networks for salience processing and executive control. J. Neurosci. 27, 2349-2356.
Seeley, W.W., Crawford, R. K., Miller, B.L. and Greicius, M. D. (2008). "Cortical neurodegeneration syndromes target human structural-functional covariance networks," in 14th International Meeting of the Organization for Human Brain Mapping Melbourne, Australia.

Sheline, Y. I., Raichle, M. E., Snyder, A. Z., Morris, J. C., Head, D., Wang, S. and Mintun, M. A. (2010). Amyloid plaques disrupt resting state default mode network connectivity in cognitively normal elderly. Biol. Psychiatry 67, 584-587.

Shimony, J. S., Zhang, D., Johnston, J. M., Fox, M. D., Roy, A., and Leuthardt, E. C. (2009). Resting-state spontaneous fluctuations in brain activity: a new paradigm for presurgical planning using fMRI. Acad. Radiol. 16, 578-583.

Shmuel, A., and Leopold, D. A. (2008). Neuronal correlates of spontaneous fluctuations in fMRI signals in monkey visual cortex: implications for functional connectivity at rest. Hum. Brain Mapp. 29, 751-761.

Shulman, R. G., Rothman, D. L., Behar, K. L., and Hyder, F. (2004). Energetic basis of brain activity: implications for neuroimaging. Trends Neurosci. 27, 489-495.

Sorg, C., Riedl, V., Muhlau, M., Calhoun, V. D., Eichele, T., Laer, L., Drzezga, A., Forstl, H., Kurz, A., Zimmer, C., and Wohlschlager, A. M. (2007). Selective changes of resting-state networks in individuals at risk for Alzheimer's disease. Proc. Natl. Acad. Sci. U.S.A. 104, 18760-18765.

Supekar, K., Menon, V., Rubin, D., Musen, M., and Greicius, M. D. (2008). Network analysis of intrinsic functional brain connectivity in Alzheimer's disease. PLoS Comput. Biol. 4, e1000100. doi: 10.1371/journal.pcbi. 1000100 .

Tian, L., Jiang, T., Wang, Y., Zang, Y., He, Y., Liang, M., Sui, M., Cao, Q., Hu, S., Peng, M., and Zhuo, Y. (2006). Altered resting-state functional connectivity patterns of anterior cingulate cortex in adolescents with attention deficit hyperactivity disorder. Neurosci. Lett. 400, 39-43.

Vanhaudenhuyse, A., Noirhomme, Q., Tshibanda, L. J., Bruno, M. A., Boveroux, P., Schnakers, C., Soddu, A., Perlbarg, V., Ledoux, D., Brichant, J. F., Moonen, G., Maquet, P., Greicius, M. D., Laureys, S., and Boly, M.(2010). Default network connectivity reflects the level of consciousness in noncommunicative brain-damaged patients. Brain 133(Pt 1), 161-171.

Vincent, J. L., Patel, G. H., Fox, M. D., Snyder, A. Z., Baker, J. T., Van Essen, D. C., Zempel, J. M., Snyder, L. H., Corbetta, M., and Raichle, M. E.
(2007). Intrinsic functional architecture in the anesthetized monkey brain. Nature 447, 83-86.

Vincent, J. L., Snyder, A. Z., Fox, M. D., Shannon, B. J., Andrews, J. R., Raichle, M. E., and Buckner, R. L. (2006). Coherent spontaneous activity identifies a hippocampal-parietal mnemonic network. J. Neurophysiol. 96, 3517-3531.

Vlieger,E. J., Majoie, C. B., Leenstra, S., and Den Heeten, G. J. (2004). Functional magnetic resonance imaging for neurosurgical planning in neurooncology. Eur. Radiol. 14, 1143-1153.

Waites, A. B., Briellman, R. S., Saling, M. M., Abbott, D. F., and Jackson, G. D. (2006). Functional connectivity networks are disrupted in left temporal lobe epilepsy. Ann. Neurol. 59, 335-343.

Wang, K., Jiang, T., Liang, M., Wang, L. Tian, L., Zhang, X., Li, K., and Liu, Z. (2006a). Discriminative analysis of early Alzheimer's disease based on two intrinsically anti-correlated networks with resting-state fMRI. Med. Image Comput. Comput. Assist. Interv. Int. Conf. Med. Image Comput. Comput. Assist. Interv. 9, 340-347.

Wang, L., Zang, Y., He, Y., Liang, M. Zhang, X., Tian, L., Wu, T., Jiang, T., and Li, K. (2006b). Changes in hippocampal connectivity in the early stages of Alzheimer's disease: evidence from resting state fMRI. Neuroimage 31, 496-504.

Wang, K., Liang, M., Wang, L., Tian, L. Zhang, X., Li, K., and Jiang, T. (2007). Altered functional connectivity in early Alzheimer's disease: a restingstate fMRI study. Hum. Brain Mapp. 28, 967-978.

Wang, L., Zhu, C., He, Y., Zang, Y., Cao, Q., Zhang, H., Zhong, Q., and Wang, Y. (2009). Altered small-world brain functional networks in children with attention-deficit/hyperactivity disorder. Hum. Brain Mapp. 30, 638-649.

Weissenbacher, A., Kasess, C., Gerstl, F., Lanzenberger, R., Moser, E., and Windischberger, C. (2009). Correlations and anticorrelations in resting-state functional connectivity MRI: a quantitative comparison of preprocessing strategies. Neuroimage 47, 1408-1416.

Weng, S. J., Wiggins, J. L., Peltier, S. J., Carrasco, M., Risi, S., Lord, C., and Monk, C. S. (2010). Alterations of resting state functional connectivity in the default network in adolescents with autism spectrum disorders. Brain Res. 1313, 202-214.

White, B. R., Snyder, A. Z., Cohen, A. L., Petersen, S. E., Raichle, M. E., Schlaggar, B. L., and Culver, J. P. (2009). Resting-state functional connectivity in the human brain revealed with dif- fuse optical tomography. Neuroimage 47, 148-156.

Whitfield-Gabrieli, S., Thermenos, H. W., Milanovic, S., Tsuang, M. T., Faraone, S. V., McCarley, R. W., Shenton, M. E., Green, A. I., NietoCastanon, A., LaViolette, P., Wojcik, J., Gabrieli, J. D., and Seidman, L. J. (2009). Hyperactivity and hyperconnectivity of the default network in schizophrenia and in first-degree relatives of persons with schizophrenia. Proc. Natl. Acad. Sci. U.S.A. 106, 1279-1284.

Wise, R. J. S., Ide, K., Poulin, M. J., and Tracey, I. (2004). Resting state fluctuations in arterial carbon dioxide induce significant low frequency variations in BOLD signal. Neuroimage 21, 1652-1664.

Yu, C., Liu, Y., Li, J., Zhou, Y., Wang, K., Tian, L., Qin, W., Jiang, T., and Li, K. (2008). Altered functional connectivity of primary visual cortex in early blindness. Hum. Brain Mapp. 29, 533-543.

Zang, Y. F., He, Y., Zhu, C. Z., Cao, Q. J., Sui, M. Q., Liang, M., Tian, L. X., Jiang, T. Z., and Wang, Y. F. (2007). Altered baseline brain activity in children with ADHD revealed by resting-state functional MRI. Brain Dev. 29, 83-91.

Zarahn, E., Aguirre, G. K., and D'Esposito, M. (1997). Empirical analyses of BOLD fMRI statistics. I. Spatially unsmoothed data collected under nullhypothesis conditions. Neuroimage 5, 179-197.

Zhang, D., Johnston, J. M., Fox, M. D., Leuthardt, E. C., Grubb, R. L. Chicoine, M. R., Smyth, M. D., Snyder, A. Z., Raichle, M. E., and Shimony, J. S. (2009a). Preoperative sensorimotor mapping in brain tumor patients using spontaneous fluctuations in neuronal activity imaged with functional magnetic resonance imaging: initial experience. Neurosurgery 65, 226-236.

Zhang, Z., Lu, G., Zhong, Y., Tan, Q., Liao, W., Chen, Z., Shi, J., and Liu, Y. (2009b). Impaired perceptual networks in temporal lobe epilepsy revealed by resting fMRI. J. Neurol. 256, 1705-1713.

Zhang, Z., Lu, G., Zhong, Y., Tan, Q., Yang, Z., Liao, W., Chen, Z., Shi, J., and Liu, Y. (2009c). Impaired attention network in temporal lobe epilepsy: a resting FMRI study. Neurosci. Lett. 458, 97-101.

Zhang, D., and Raichle, M. E. (2010). Disease and the brain's dark energy. Nat. Rev. Neurol. 6, 15-28.

Zhang, D., Snyder, A. Z., Fox, M. D., Sansbury, M. W., Shimony, J. S., and Raichle, M. E. (2008). Intrinsic functional relations between human cerebral cortex and thalamus. J. Neurophysiol. 100, 1740-1748. 
Zhou, Y., Liang, M., Jiang, T., Tian, L., Liu, Y., Liu, Z., Liu, H., and Kuang, F. (2007). Functional dysconnectivity of the dorsolateral prefrontal cortex in first-episode schizophrenia using resting-state fMRI. Neurosci. Lett. 417, 297-302.

Zhu, C. Z., Zang, Y. F., Cao, Q. J., Yan, C. G., He, Y., Jiang, T. Z., Sui, M. Q., and Wang, Y. F. (2008). Fisher discriminative analysis of resting-state brain function for attention-deficit/hyperactivity disorder. Neuroimage 40, 110-120.
Zhu, C.Z., Zang, Y. F., Liang, M., Tian, L.X., He, Y., Li, X. B., Sui, M. Q., Wang, Y. F., and Jiang, T. Z. (2005). Discriminative analysis of brain function at resting-state for attention-deficit/hyperactivity disorder.Med. Image Comput. Comput. Assist. Interv. Int. Conf. Med. Image Comput. Comput. Assist. Interv. 8, 468-475.

Zuo,X.N., Kelly, C., Adelstein, J.S., Klein,D. F., Castellanos, F.X., and Milham, M.P. (2009). Reliable intrinsic connectivity networks: test-retest evaluation using
ICA and dual regression approach. Neuroimage 49, 2163-2177.

Conflict of Interest Statement: The authors declare that the research was conducted in the absence of any commercial or financial relationships that could be construed as a potential conflict of interest.

Received: 09 February 2010; paper pending published: 11 March 2010; accepted: 11 May 2010; published online: 17 June 2010.
Citation: Fox MD and Greicius M (2010) Clinical applications of resting state functional connectivity. Front. Syst. Neurosci. 4:19. doi: 10.3389/fnsys.2010.00019

Copyright $\odot 2010$ Fox and Greicius. This is an open-access article subject to an exclusive license agreement between the authors and the Frontiers Research Foundation, which permits unrestricted use, distribution, and reproduction in any medium, provided the original authors and source are credited. 